\title{
Modulated rotating convection: radially travelling concentric rolls
}

\author{
A. RUBIO ${ }^{1}$, J. M. LOPEZ1 AND F. MARQUES ${ }^{2}$ \\ ${ }^{1}$ Department of Mathematics and Statistics, Arizona State University, Tempe, AZ 85287, USA \\ ${ }^{2}$ Departament de Física Aplicada, Univ. Politècnica de Catalunya, Barcelona 08034, Spain
}

(Received 11 September 2007 and in revised form 1 May 2008)

Recent experiments in rotating convection have shown that the spatio-temporal bulk convective state with Küppers-Lortz dynamics can be suppressed by small-amplitude modulations of the rotation rate. The resultant axisymmetric pulsed target patterns were observed to develop into axisymmetric travelling target patterns as the modulation amplitude and Rayleigh number were increased. Using the Navier-StokesBoussinesq equations with physical boundary conditions, we are able to numerically reproduce the experimental results and gain physical insight into the responsible mechanism, relating the onset of the travelling target patterns to a symmetry-restoring saddle-node on an invariant circle bifurcation. Movies are available with the online version of the paper.

\section{Introduction}

It is well known that temporal forcing in dynamical systems can stabilize states that are otherwise unstable, as well as producing new phenomena not present in the unforced problem (Davis 1976). Recent examples in fluid dynamics include TaylorCouette flow with axial harmonic oscillations of the inner cylinder that substantially shift the transition to Taylor vortices to faster rotation rates of the inner cylinder (Weisberg, Kevrekidis \& Smits 1997; Marques \& Lopez 1997). In that problem, when the transition does occur, it takes place in a complex catastrophic way (Sinha, Kevrekidis \& Smits 2006; Avila et al. 2007). Another striking example is vortex breakdown in a cylindrical container driven by the rotation of one endwall. In that case, the oscillations of the vortex breakdown bubbles are quenched by a smallamplitude harmonic modulation of the rotation (Lopez et al. 2008). These are all examples of how the oscillatory Stokes layers produced by the harmonic modulations inhibit instabilities of the unmodulated state.

Rayleigh-Bénard convection has been, and continues to be, a very popular hydrodynamic system in which to study the effects of temporal forcing (Davis 1976; Bodenschatz, Pesch \& Ahlers 2000). The early studies either modulated the vertical acceleration or the temperature applied at a horizontal boundary, and were primarily concerned with shifts in the threshold for the onset of convection due to the modulation. Both of these modes of modulation suffer from experimental limitations in their implementation (Bodenschatz et al. 2000) as well as theoretical issues arising from the forced breaking of the mid-plane reflection symmetry, commonly referred to as the Boussinesq symmetry (Roppo, Davis \& Rosenblat 1984).

Rotating Rayleigh-Bénard convection (RRBC) in a cylinder, where the rotation rate is modulated, has been studied theoretically (Bhattacharjee 1990) and experimentally (Niemela, Smith \& Donnelly 1991) with regards to threshold shifts. Thompson, 
Bajaj \& Ahlers (2002) experimentally observed synchronous spiral and axisymmetric pulsed target patterns (the observed type of pattern depending on experimental protocols) at low modulation amplitudes of the rotation rate in parameter regimes where the unmodulated state manifested spatio-temporal Küppers-Lortz (KL) dynamics (Küppers \& Lortz 1969). At larger modulation amplitudes, the axisymmetric pulsed target patterns undergo transition to a modulated travelling target pattern in which the innermost convective roll collapses in on itself and a new convective roll develops at larger radii as the entire pattern drifts radially inward. The target and spiral patterns have been qualitatively computed using a model based on the Swift-Hohenberg equation (Roxin \& Riecke 2002). The model essentially treated the problem as a two-dimensional pattern-forming system, neglecting its vertical structure. The qualitative nature of the model precluded quantitative comparisons with the experiments. Furthermore, the radially travelling target patterns could not be simulated using that model and the origin of the radial drift remained unidentified.

Similar radially travelling target patterns have been simulated using the NavierStokes-Boussinesq equations in axisymmetric subspaces of both non-rotating (Tuckerman \& Barkley 1988) and rotating (Lopez, Rubio \& Marques 2006) RayleighBénard convection in cylinders. In both cases, the travelling target patterns were found to originate at a SNIC (saddle-node on an invariant circle) bifurcation. Here, we have simulated the conditions corresponding to the experiments of Thompson et al. (2002) using the Navier-Stokes-Boussinesq equations and have reproduced the various observed states, including the radially travelling target patterns. By considering how the period of the travelling target pattern varies with the forcing amplitude and the Rayleigh number, we show that the travelling target patterns in the modulated rotation problem also arise via a SNIC bifurcation in which the Boussinesq symmetry plays a central role. Furthermore, it is apparent that it is not the modulated rotation that is responsible for the radial drift, but rather the restriction of the dynamics to an axisymmetric subspace in which the SNIC bifurcation of pulsed target patterns leads to the radially travelling circular rolls.

The nature of this problem captures the competition and nonlinear interactions between thermal convective instabilities and the large-scale circulation mechanically driven by the modulated rotation of the cylinder. In the absence of modulation, for large enough Rayleigh number, the base state consists of Küppers-Lortz spatiotemporal chaos. On the other hand, for small Rayleigh number, the base state consists of the oscillatory axisymmetric large-scale circulation originating in the Stokes layers at the top and bottom boundaries. When both mechanisms are of comparable strength, the numerical simulations show that the Küppers-Lortz chaotic state is replaced by pulsed and travelling target patterns, in agreement with the aforementioned experimental results.

\section{Governing equations, symmetries and numerical technique}

\subsection{Governing equations}

Consider the flow in a circular cylinder, with no-slip boundary conditions, of radius $r_{0}$ and depth $d$, with a modulated rotation $\omega\left(t^{*}\right)=\omega_{0}+\omega_{1} \sin \left(\omega_{m} t^{*}\right)$, where $t^{*}$ is dimensional time in seconds. The endwalls are maintained at constant temperatures, $T_{0}-\Delta T / 2$ at the top and $T_{0}+\Delta T / 2$ at the bottom, and the sidewall is insulating. The Boussinesq approximation is implemented, treating all fluid properties as constant except for the density in the gravitational term, which varies linearly with temperature. The centrifugal buoyancy (Lopez et al. 2006; Marques et al. 2007) is ignored in this 
paper since in the experiments (Thompson et al. 2002), the Froude number was very small $\left(F r=\omega_{0}^{2} r_{0} / g<0.016\right.$, where $r_{0}$ is the cylinder radius, $\omega_{0}$ is the mean rotation rate and $g$ is the acceleration due to gravity. The system is non-dimensionalized using $d$ as the length scale, $d^{2} / v$ as the time scale ( $v$ is the kinematic viscosity), $v^{2} \rho_{0} / d^{2}$ as the pressure scale $\left(\rho_{0}\right.$ is the density at mean temperature $T_{0}$ ), and $\Delta T$ (the difference in temperature between the top and bottom) as the temperature scale. In a frame of reference rotating at the mean rotation rate $\omega_{0}$, the non-dimensional governing equations are

$$
\begin{aligned}
\left(\partial_{t}+\boldsymbol{u} \cdot \nabla\right) \boldsymbol{u}= & -\nabla P+\nabla^{2} \boldsymbol{u}+\frac{R a}{\sigma}(\Theta-z) \hat{z}+2 \Omega_{0} \boldsymbol{u} \times \hat{z}-\frac{R a F r}{\sigma \gamma}(\Theta-z) r, \\
& \left(\partial_{t}+\boldsymbol{u} \cdot \nabla\right) \Theta=w+\sigma^{-1} \nabla^{2} \Theta, \quad \nabla \cdot \boldsymbol{u}=0,
\end{aligned}
$$

where $\boldsymbol{u}=(u, v, w)$ is the velocity field in cylindrical coordinates $(r, \theta, z), P$ is the dynamic pressure and $\hat{z}$ is the vertical unit vector in the $z$-direction. As noted above, $F r=0$ in this study. $\Theta$ is the temperature deviation with respect to the conductive linear temperature profile; the relationship between $\Theta$ and the temperature $T$ (both non-dimensional) is given by

$$
T=T_{0} / \Delta T-z+\Theta,
$$

where $T_{0} / \Delta T-z$ is the conductive temperature profile.

There are six non-dimensional parameters:

$$
\begin{array}{ll}
\text { Rayleigh number : } & R a=\alpha g d^{3} \Delta T / \kappa \nu, \\
\text { Coriolis number : } & \Omega_{0}=\omega_{0} d^{2} / \nu, \\
\text { Prandtl number : } & \sigma=v / \kappa, \\
\text { aspect ratio : } & \gamma=r_{0} / d, \\
\text { modulation amplitude : } & \Omega_{1}=\omega_{1} d^{2} / \nu, \\
\text { modulation frequency : } & \Omega_{m}=\omega_{m} d^{2} / \nu,
\end{array}
$$

where $\alpha$ is the coefficient of volume expansion, and $\kappa$ is the thermal diffusivity. The boundary conditions (in a frame of reference rotating at the mean rotation rate $\omega_{0}$ ) are:

$$
\begin{array}{ll}
r=\gamma: & \Theta_{r}=u=w=0, v=\gamma \Omega(t), \\
z= \pm 0.5: & \Theta=0, u=w=0, v=r \Omega(t),
\end{array}
$$

where $\Omega(t)=\Omega_{1} \sin \left(\Omega_{m} t\right)$ is the angular velocity of the container in the reference frame rotating with the mean angular velocity $\Omega_{0}$. To simplify the discussion, we introduce the relative modulation amplitude $A=\Omega_{1} / \Omega_{0}$ to allow for comparison between states with differing $\Omega_{0}$. A will be used instead of $\Omega_{1}$, except in the particular case when $\Omega_{0}=0$, as then $A$ is not defined.

\subsection{Symmetries}

The governing equations and boundary conditions are invariant under arbitrary rotations about the axis, $R_{\alpha}$, whose action is

$$
R_{\alpha}(u, v, w, \Theta)(r, \theta, z, t)=(u, v, w, \Theta)(r, \theta+\alpha, z, t) .
$$

They are also reflection-symmetric about the cylinder half-height. The action $K_{z}$ of this so-called Boussinesq symmetry is

$$
K_{z}(u, v, w, \Theta)(r, \theta, z, t)=(u, v,-w,-\Theta)(r, \theta,-z, t) .
$$


The symmetry group of the system is $\mathscr{G}=S O(2) \times Z_{2}$, with $S O(2)$ generated by $R_{\alpha}$ and $Z_{2}$ by $K_{z}$. The basic state is $\mathscr{G}$-invariant and $\tau_{m}$-periodic, where $\tau_{m}=2 \pi / \Omega_{m}$.

In the absence of any rotation $\left(\Omega_{0}=\Omega_{1}=0\right)$, the system is also invariant under reflections on meridional planes ( $\theta$ constant), that together with the invariance to rotations about the axis generate the $O(2)=S O(2) \rtimes Z_{2}$ symmetry group; the $Z_{2}$ component is generated by the reflection (angle flip) symmetry

$$
K_{\theta}(u, v, w, \Theta)(r, \theta, z, t)=(u,-v, w, \Theta)(r,-\theta, z, t) .
$$

The symbol $\rtimes$ indicates the semidirect product of the symmetry groups; it is not the direct product $(\times)$ because the flip symmetry $K_{\theta}$ does not commute with axial rotations $R_{\alpha}: K_{\theta} R_{\alpha}=R_{-\alpha} K_{\theta}$. The symmetry group of the system in the absence of any rotation is $\mathscr{G}=O(2) \times Z_{2}$. Note further that when the modulated rotation has zero mean $\left(\Omega_{0}=0\right.$, but $\left.\Omega_{1} \neq 0\right)$, the angle flip $K_{\theta}$ is no longer a symmetry of the problem, but the system is still invariant under a space-time symmetry $H$, whose action is the composition of $K_{\theta}$ with an evolution of half the modulation period:

$$
H(u, v, w, \Theta)(r, \theta, z, t)=(u,-v, w, \Theta)\left(r,-\theta, z, t+\tau_{m} / 2\right) .
$$

In particular, when restricting the $\Omega_{0}=0$ problem to the axisymmetric subspace, the symmetry group of the system in the zero mean modulation case is $Z_{2} \times Z_{2}$, generated by $H$ and $K_{z}$. Although in this paper we consider only non-zero mean rotations, $\Omega_{0} \neq 0$, some of the solutions obtained exhibit characteristics of the symmetry $H$ being slightly broken, since the values of $\Omega_{0}$ considered are relatively small. Finally, it is useful to introduce a global Poincare map which is the stroboscopic map of the periodically forced system

$$
P(u, v, w, \Theta)\left(r, \theta, z, t_{0}\right) \mapsto(u, v, w, \Theta)\left(r, \theta, z, t_{0}+\tau_{m}\right) .
$$

For any function of time $f(t)$, we can analogously define the action of $P$ on it as $(P f)\left(t_{0}\right)=f\left(t_{0}+\tau_{m}\right)$; the time phase $t_{0}$ can be chosen arbitrarily, resulting in different Poincare maps, all of them equivalent. To be specific, in this paper we will use the phase $t_{0}=0$. For any variable $f(t)$, the discrete orbit generated by $P$, starting at $f(0)$, is given by $P^{n} f=f\left(n \tau_{m}\right)$; it is obtained by strobing $f(t)$ at the beginning of each forcing period.

To describe the heat transfer properties of a solution we use the Nusselt number, the ratio between the heat transfer of the solution considered, and the heat transfer of the conductive state, both through the top lid. It is given by the negative average of the derivative of the temperature field at the top lid,

$$
N u=-\left.\langle\partial T / \partial z\rangle\right|_{z=0.5}=\left.\langle 1-\partial \Theta / \partial z\rangle\right|_{z=0.5} .
$$

We could also compute the heat transfer at the bottom lid, $N u^{b}=\left.\langle\partial T / \partial z\rangle\right|_{z=-0.5}$. The $K_{z}$ reflection relates $N u$ and $N u^{b}$; the relationship depends on how the solution considered transforms under $K_{z}$. The breaking of $K_{z}$-symmetry will be one of the focuses of this paper, resulting in pairs of $K_{z}$-symmetry related states. Integrating the temperature equation (2.2) in the fluid domain and using the divergence theorem and the boundary conditions of the problem, the following relationship between both Nusselt numbers is obtained,

$$
N u^{b}-N u=\sigma \frac{\mathrm{d}}{\mathrm{dt}}\langle T\rangle_{V},
$$

where \langle\rangle$_{V}$ means the volume average in the fluid domain. For steady solutions, the time derivative is zero, and the Nusselt-numbers coincide: $N u=N u^{b}$; therefore, 
$K_{z}$-related steady solutions must have the same Nusselt-number value. For periodic solutions, both Nusselt numbers are time-dependent; their instantaneous values are not necessarily the same, but their time-averaged values are still identical (because the average of the time derivative in (2.10) is zero). Therefore, $K_{z}$-related periodic solutions have different instantaneous Nusselt-number values, and so different $P^{n}(N u)$ values. However, their time-averaged values are the same. For periodically forced flows, as in the present problem, it is very convenient to use the Nusselt number averaged over a forcing period, $\overline{N u}$.

\subsection{Numerical technique}

The governing equations have been solved using the second-order time-splitting method proposed in Hugues \& Randriamampianina (1998) combined with a pseudospectral method for the spatial discretization, using a Galerkin-Fourier expansion in the azimuthal coordinate $\theta$ and Chebyshev collocation in $r$ and $z$. In order to handle the coordinate singularity at the axis due to the use of polar coordinates, and avoid unnecessary clustering of grid points near the axis, the prescription in Fornberg (1998) has been followed. The expansion for the thermal perturbation is given by

$$
\Theta(r, \theta, z)=\operatorname{Re} \sum_{m=0}^{2 n_{r}+1} \sum_{n=0}^{n_{z}} \sum_{k=-n_{\theta} / 2}^{n_{\theta} / 2-1} \Theta_{m n k} T_{m}(r / \gamma) T_{n}(2 z) \mathrm{e}^{\mathrm{i} k \theta} .
$$

The expansions for the velocity components $u, v$ and $w$ are analogous. Fornberg's prescription is that $\Theta_{m n k}=w_{m n k}=0$ when $k+m$ is odd, and $u_{m n k}=v_{m n k}=0$ when $k+m$ is even; there are precisely $n_{r}+1$ non-zero coefficients for fixed $n$ and $k$ in (2.11). Owing to the expansion (2.11) the origin is never a collocation point, and the equations are solved in the physical domain $(r, \theta, z)=(0, \gamma] \times(0,2 \pi] \times[-1 / 2,1 / 2]$. Following Orszag \& Patera (1983), we have used the combinations $u_{+}=u+\mathrm{i} v$ and $u_{-}=u-\mathrm{i} v$ in order to decouple the linear diffusion terms in the momentum equations. For each Fourier mode, the resulting Helmholtz equations for $\Theta, w, u_{+}$ and $u_{-}$have been solved using a diagonalization technique in the two coordinates $r$ and $z$. Fornberg's prescription guarantees the regularity conditions at the origin required in order to solve the Helmholtz equations (Mercader, Net \& Falqués 1991).

We have tested the code on a number of convection problems in rotating cylinders (Lopez et al. 2006, 2007; Marques et al. 2007); establishing resolution requirements over a wide range of parameter regimes. For the problems considered in this paper, we have used $n_{r}=64$ and $n_{z}=24$ Chebyshev modes. For cases where the solution is non-axisymmetric, we have used up to $n_{\theta}=184$ Fourier modes. We have used time steps $\delta t \leqslant 10^{-3}$ viscous time units (depending on the modulation frequency). We have fixed the aspect ratio $\gamma=11.8$ and Prandtl number $\sigma=4.5$ to correspond to those in the experiments of Thompson et al. (2002), and consider variations in $A$ and $\Omega_{m}$ for selected values of $R a$ and $\Omega_{0}$ in the range considered in the experiments. To simulate the experimentally observed axisymmetric patterns, we restrict our computations to the axisymmetric subspace. This restriction affords a detailed exploration of the dynamics over an extensive range of parameter space.

\section{Results}

We begin by briefly describing the unmodulated RRBC state which is being quenched by the modulated rotation. It is a state of spatio-temporal complexity which arises essentially directly from the conduction state as $R a$ is increased (for the rotation rates considered here). 


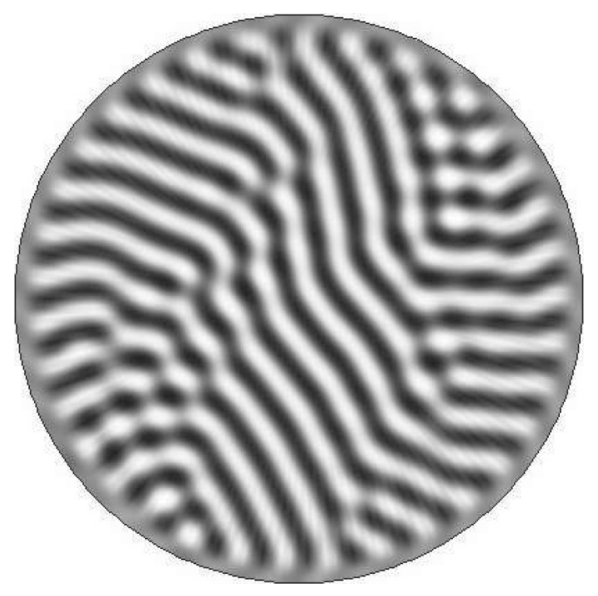

FIGURE 1. Snap-shot of isotherms at mid-height of a Küppers-Lortz state at $R a=2868.8$, $\Omega_{0}=23.6, \gamma=11.8$ and $A=0$. Movie 1 shows the spatio-temporal characteristics of the isotherms at mid-height of this state over 450 viscous times at a rate of 20 frames per second, with each frame being 0.45 viscous times apart.

\subsection{Unmodulated RRBC: Küpper-Lortz state}

In the absence of modulation $(A=0)$, for $R a$ less than a critical value $R a_{c}$ that depends on $\Omega_{0}, \gamma$ and $\sigma$, the system is in a state of solid-body rotation with a linear temperature profile in $z$, i.e. the trivial conduction state. For $R a>R a_{c}$, KL spatio-temporal chaos is observed. This consists of patterns of convection rolls that break up into domains of various orientations with propagating fronts between the domains, and defects that nucleate at the cylinder wall evolving into growing patches of rolls oriented at angles different from the rolls nearby in the interior, all occurring with temporal irregularity. Figure 1 shows computed isotherms at mid-height of a KL state at $R a=2868.8$ and $\Omega_{0}=23.6$, corresponding to the experimental conditions of the KL state in figure 1(a) of Thompson et al. (2002). Movie 1, available with the online version of the paper, shows the spatio-temporal characteristics of the isotherms at mid-height of this state over 450 viscous times at a rate of 20 frames per second, with each frame being 0.45 viscous times apart. This state is typical for the range of $R a$ and $\Omega_{0}$ considered in this paper when $A=0$. The associated spatio-temporal chaos has been the subject of extensive experimental and amplitude equation investigations (see Knobloch 1998; Bodenschatz et al. 2000, and references therein), and more recently it has also been studied numerically using the Navier-Stokes-Boussinesq equations with no-slip boundary conditions (Sánchez-Álvarez et al. 2005; Scheel \& Cross 2005; Becker et al. 2006).

\subsection{Modulated basic state}

In the modulated problem $(A \neq 0)$, there is no trivial conduction state for any $R a \neq 0$ as the modulated rotation induces a mechanically driven large-scale flow. Without modulation, for $R a<R a_{c}\left(\Omega_{0}, \gamma, \sigma\right)$, the flow is in a state of solid-body rotation with a linear temperature profile vertically across the layer $(\Theta=0)$. For any modulation, $A \neq 0$, Stokes layers (analogues of the boundary layer on a plate oscillating in its own plane; see Stokes 1851; Batchelor 1967; Kerczek \& Davis 1974; Davis 1976; Yih 1977) form on the top and bottom boundaries, whose thicknesses scale with $1 / \sqrt{\Omega_{m}}$. These Stokes layers arise as the vortex lines, which for $A=0$ are parallel to the rotation axis, are displaced in the layers radially outward (inward) during the acceleration (deceleration) phase of the modulation. This bending of the vortex lines in the Stokes 
0

0.02

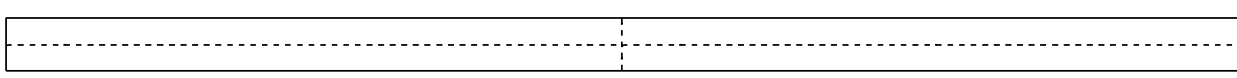

0.04

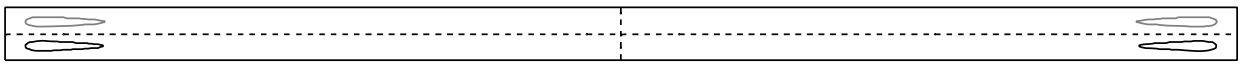

0.06

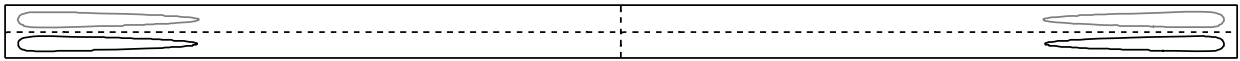

0.08

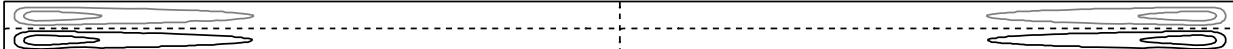

0.10

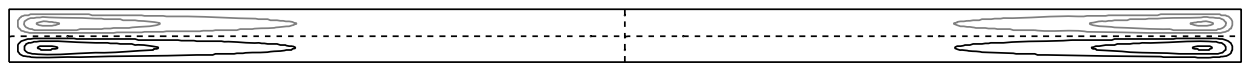

0.12

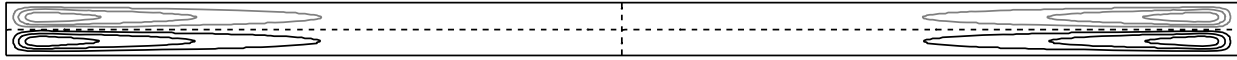

0.14

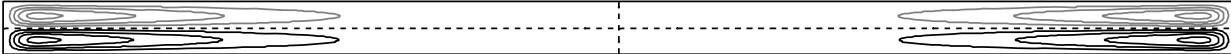

0.16

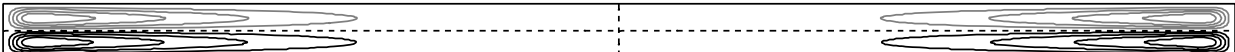

0.18

$\Leftrightarrow$

0.20

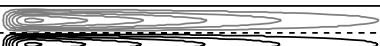

CO

Figure 2. Snap-shots in a meridional plane $(r \in[-\gamma, \gamma], z \in[-0.5,0.5])$ of the streamfunction of basic states, all at the same phase of the imposed modulation, at $R a=2600$, $\Omega_{0}=\Omega_{m}=23.6$ and indicated $A$. Five contours are spaced linearly with $\Psi \in[-5,5]$; black (grey) contours are negative (positive) and the zero contour is dashed.

layers gives rise to a secondary flow which centrifuges fluid in the layers radially outward (inward) with the acceleration (deceleration) of the rotation of the cylinder. The modulated rotation also drives fluid into and out of the Stokes layers from the interior (the associated Ekman pumping phenomenon). However, the major impact on the interior flow comes from the fluid in the top and bottom Stokes layers being deflected into the sidewall layer and forming a radial jet at mid-height $(z=0)$. This radial jet injects fluid with high angular momentum (acquired in the Stokes layers) into the interior at mid-height during the acceleration phase. During the deceleration phase, the fluid flows in the opposite direction: fluid from the interior is sucked into the sidewall layer and transported into the top and bottom Stokes layers. In figure 2, we show the development of the Stokes layers and sidewall jet with increasing $A$ at $R a=2600$ with $\Omega_{0}=\Omega_{m}=23.6$, at a fixed phase of the modulation (during the acceleration phase, when the radial outflow in the Stokes layer is greatest). For small $A$, the actions of the Stokes layers and sidewall jet are restricted to the vicinity of the sidewall. As $A$ is increased, this action extends deeper in toward the axis, roughly as $\sqrt{A}$. The flow in the centre of the cell remains essentially in solid-body rotation out to a radius that depends on both $A$ and $\Omega_{m}$.

Figure 3 illustrates the hydrodynamics of the modulated basic state in the neighbourhood of the sidewall, $r \in[0.8 \gamma, \gamma]$, for $R a=2600, \Omega_{0}=\Omega_{m}=23.6$ and $A=0.05$ (under these conditions, the basic state is stable). Shown in the first column of the figure is the vortex line bending driving the secondary flow and sidewall jet (illustrated by the radial velocity in the second column, and instantaneous streamlines in the third column), which drive a time-dependent temperature perturbation (shown 
Time $r v+\Omega_{0} r^{2}$

$u$

$\Psi$

$\Theta$
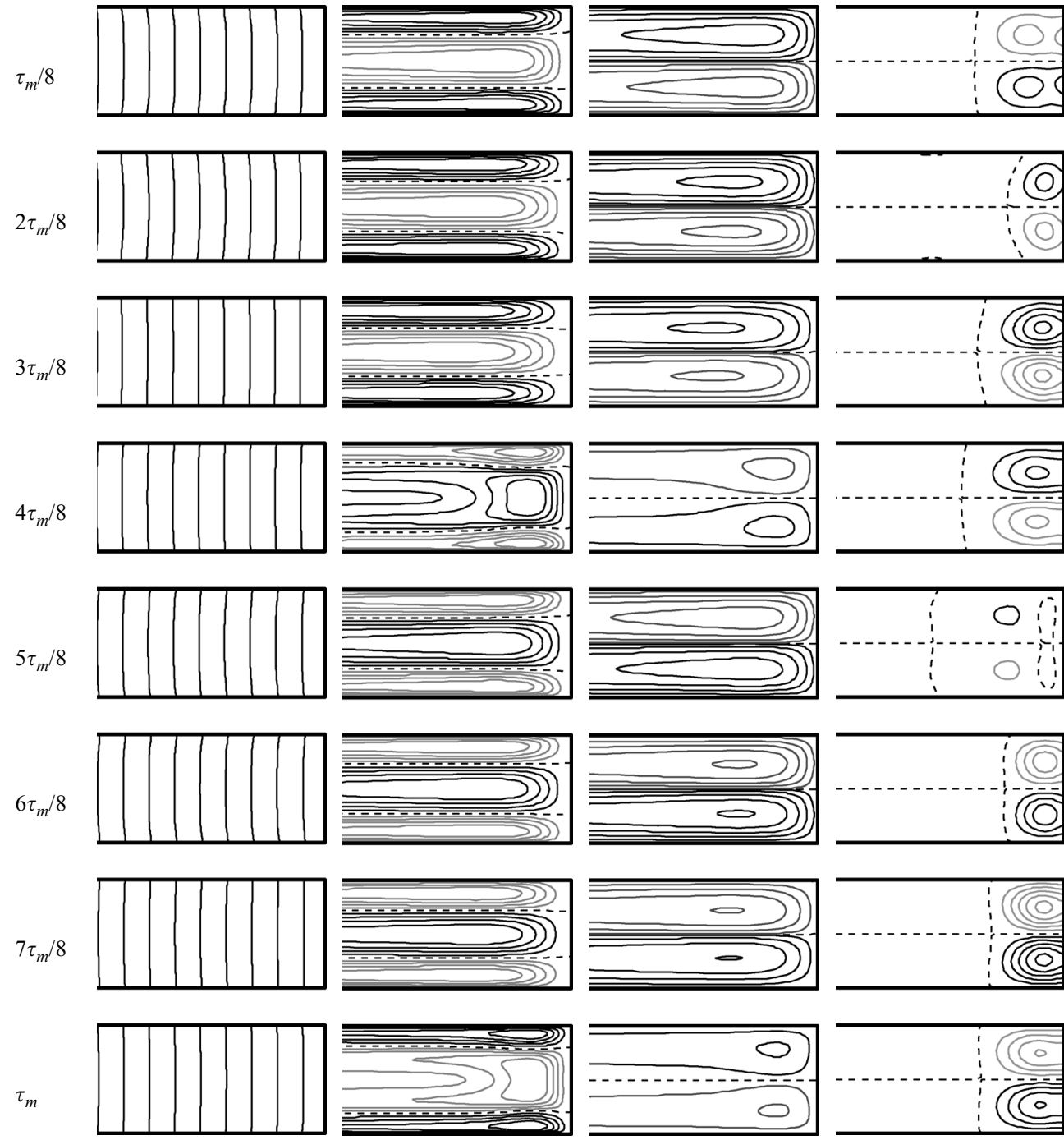

FIGURE 3. Contours of the angular momentum $r v+\Omega_{0} r^{2}$, radial velocity $u$, streamfunction $\Psi$, and the temperature deviation $\Theta$ in a part of a meridional plane near the sidewall $(r \in[0.8 \gamma, \gamma], z \in[-0.5,0.5])$ over one forcing period $\tau_{m}=2 \pi / \Omega_{m}$, for a modulated basic state at $R a=2600, \Omega_{0}=\Omega_{m}=23.6$ and $A=0.05$. Five contour levels are linearly spaced for $\Theta \in[-0.025,0.025]$ and $u \in[-10,10]$, and five contour levels are quadratically spaced for $\Psi \in[-1.25,1.25]$ and ten contours are quadratically spaced for $r v+\Omega_{0} r^{2} \in[9500,14250]$.

in the fourth column). This temperature deviation, consisting of relatively hot and cold axisymmetric rolls at the lower and upper corners of the sidewall, alternating synchronously with the forcing, exists for all $R a$ when $A \neq 0$. Their presence leads to a heat flux across the layer enhanced from that due purely to conduction, i.e. for any $R a>0$ and $A>0$, the Nusselt number is $N u>1$.

The modulated basic state is both axisymmetric and $K_{z}$-reflection symmetric about $z=0$, i.e. the symmetry group of the basic state is $S O(2) \times Z_{2}$, and it is synchronous with the modulation frequency. The usual $N u>1$ criterion for the onset of thermal 

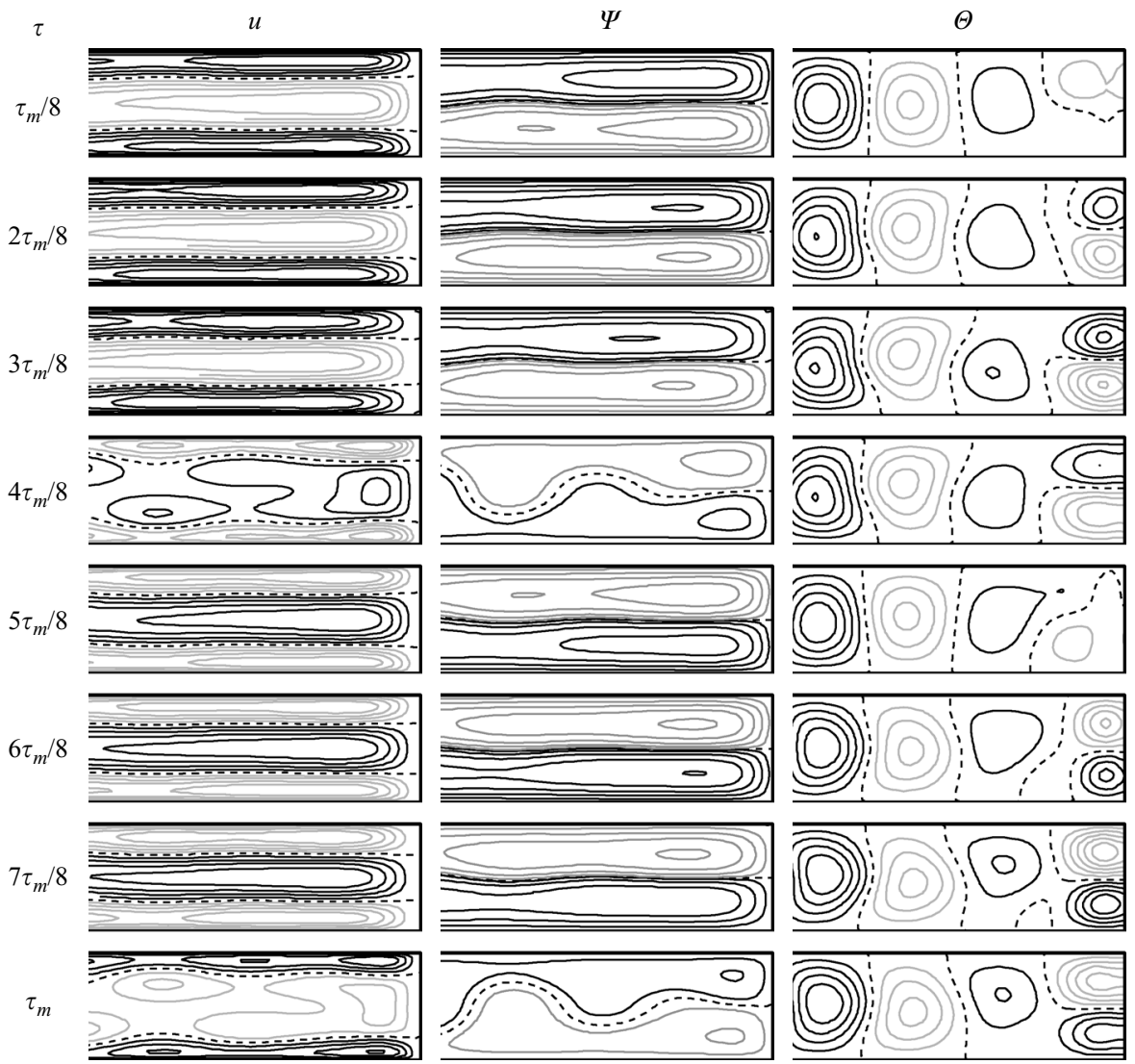

Figure 4. Contours of the radial velocity $u$, streamfunction $\Psi$ and temperature deviation $\Theta$ in a meridional plane $(r \in[0.74 \gamma, \gamma], z \in[-0.5,0.5])$ over one forcing period of the pulsed target pattern at $R a=2700, \Omega_{0}=\Omega_{m}=23.6$, and $A=0.05$. Five contours are quadratically spaced for $\Psi \in[-1.25,1.25]$ and are linearly spaced for $\Theta \in[-0.025,0.025]$ and $u \in[-10,10]$.

convection cannot be used to determine the instability of the basic state. Instead, we detect the onset of axisymmetric thermal convection by monitoring the temperature on the axis at mid-height $\Theta_{m}(t)=\Theta(r=0, \theta=0, z=0, t)$. For the basic state, $\Theta_{m}=0$, and the onset of axisymmetric convection breaks the Boussinesq reflection symmetry $K_{z}$, giving states with $\Theta_{m} \neq 0$.

\subsection{Onset of thermal convection: axisymmetric pulsed target patterns}

For a given $A \neq 0$, on increasing $R a$ beyond $R a_{c}=2627$, the modulated basic state loses stability via a pitchfork bifurcation that breaks the Boussinesq reflection symmetry $K_{z}$. At this bifurcation, a pair of states synchronous with the modulation period emerge. Near the sidewall, the hydrodynamics of these bifurcating pulsed target patterns is very similar to that of the modulated basic state (illustrated in figure 3), although the symmetry $K_{z}$ has been broken by the onset of convection, as can be seen in figure 4 . This symmetry breaking is characterized by the development of a thermal plume at the axis, which comes in two varieties: one is a hot plume rising and the other is a cold plume descending on the axis. These plumes can be seen in figures 5 and 6 , which show snap-shots at increasing $R a$ of $\Theta$-isotherms in a meridional plane, at a fixed phase of the modulation, for $\Omega_{0}=\Omega_{m}=23.6$ and $A=0.18$ 


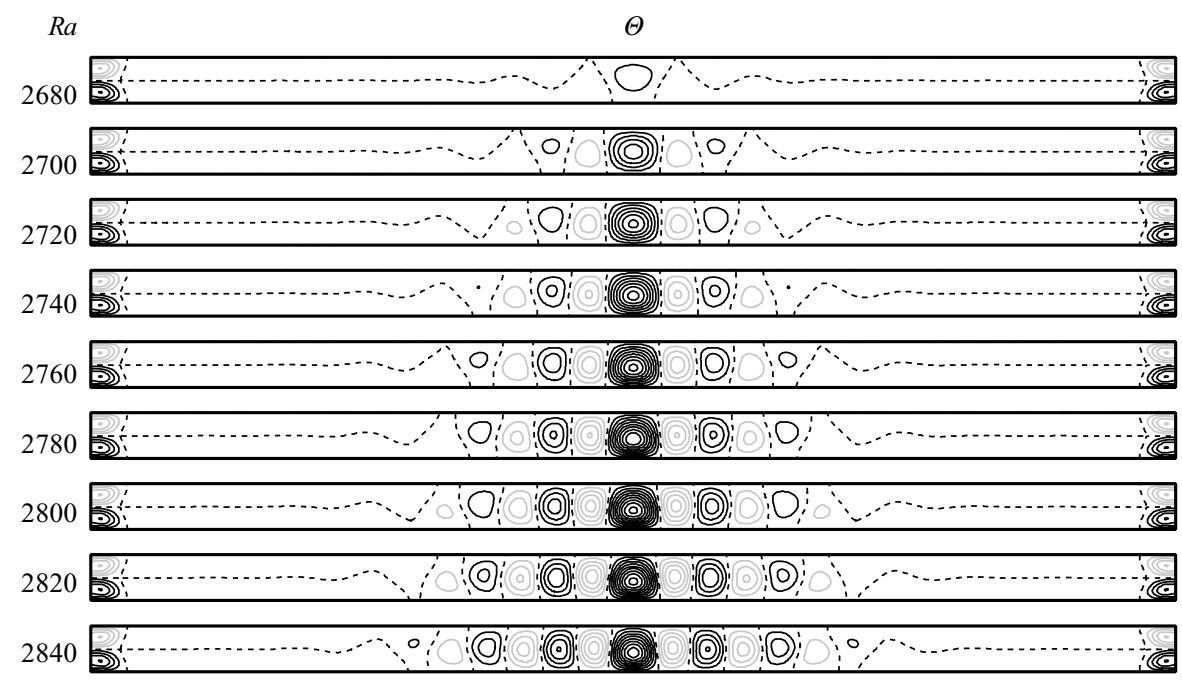

FIGURE 5. Snap-shots in a meridional plane of $\Theta$ for pulsed target states, all at the same phase of the imposed modulation, at $A=0.18$ and $\Omega_{0}=\Omega_{m}=23.6$ for $R a$ as indicated. Ten contours are evenly spaced with $\Theta \in[-0.5,0.5]$.

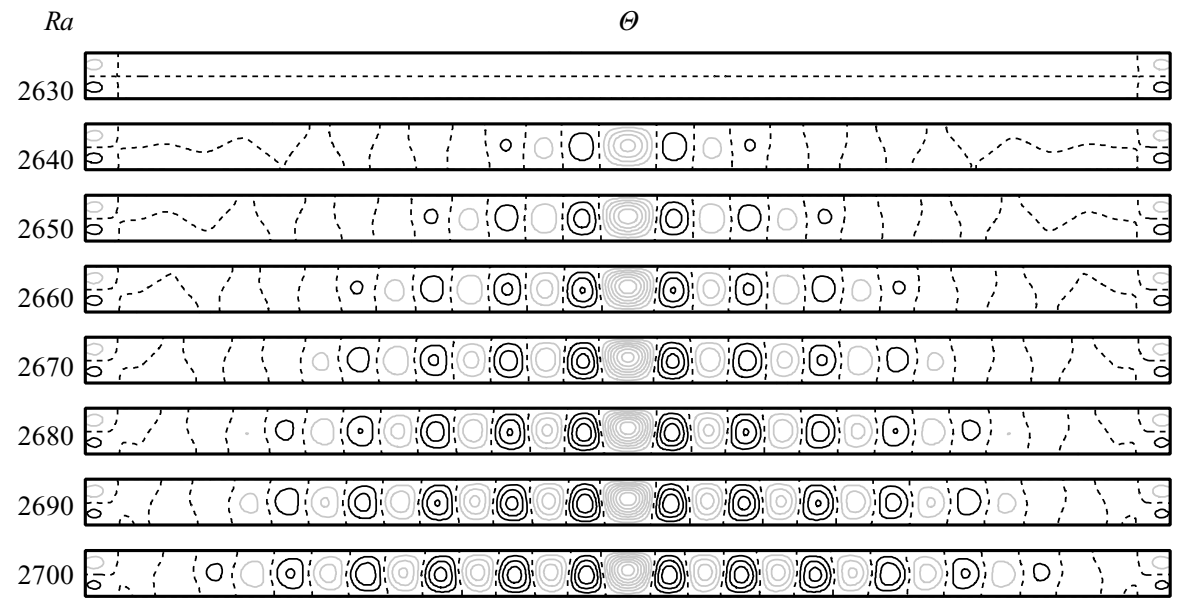

FIGURE 6. Snap-shots in a meridional plane of $\Theta$ for pulsed target states, all at the same phase of the imposed modulation, at $A=0.05$ and $\Omega_{0}=\Omega_{m}=23.6$ for $R a$ as indicated. Ten contours are evenly spaced with $\Theta \in[-0.5,0.5]$.

and 0.05 , respectively, illustrating the development of the pulsed target patterns. With increasing $R a$, concentric rolls develop, growing out from the axis; the sidewall jet displaces the nearby rolls alternately closer to and further from the axis of rotation with no net movement over the course of a forcing period (figure 4). The isotherms corresponding to the symmetrically related branch correspond to reflecting those in figures 5 and 6 about the mid-height $z=0$ and changing the sign of $\Theta$, i.e. applying the action of $K_{z}$. Figure 7 shows snap-shots of the isotherms at the mid-plane $z=0$ of two target states with warm cores at $\Omega_{0}=\Omega_{m}=23.6$, and $R a=2840, A=0.18$ and $R a=2700, A=0.05$. The synchronous pulsation and lack of net radial movement is 
(a)

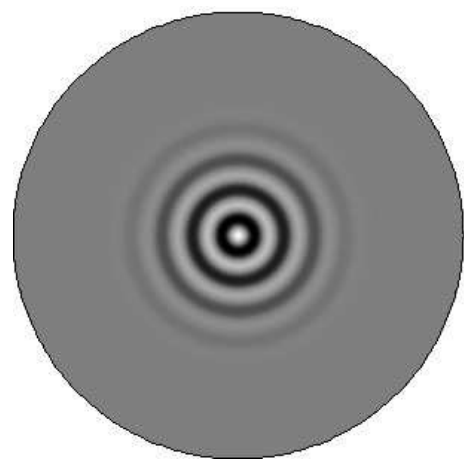

(b)

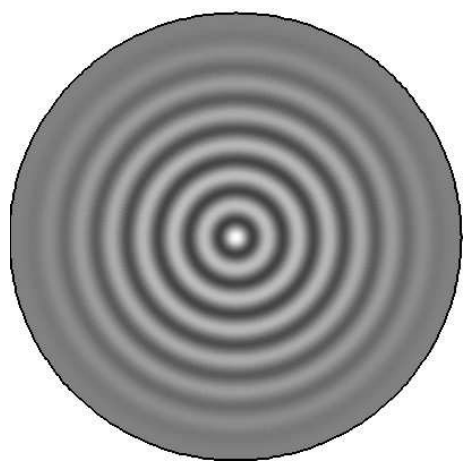

FIGURE 7. Snap-shots of isotherms at mid-height for axisymmetric pulsed target patterns at (a) $R a=2840$ and $A=0.18$, and at (b) $R a=2700$ and $A=0.05$, both with $\Omega_{0}=\Omega_{m}=23.6$ (ten forcing periods of the $R a=2700$ pattern are shown in movie 2 .



FigURE 8. Snap-shots in a meridional plane of streamfunction for pulsed target states, all at the same phase of the imposed modulation, at $R a=2680, \Omega_{0}=\Omega_{m}=23.6$ and $A$ as indicated. Ten contours are spaced quadratically with $\Psi \in[-5,5]$.

illustrated in movie 2 available with the online version of the paper, which shows the pulsed target pattern state from figure $7(b)$ over ten modulation periods.

The development with $A$ of the radial velocity of pulsed target patterns at $\Omega_{0}=\Omega_{m}=23.6$ and $R a=2680$ is illustrated in figure 8. The state at $A=0$ is the steady target pattern of the axisymmetric subspace of the unmodulated problem (which is unstable to the KL state). It is evident that the action of the oscillatory Stokes layers is stronger for larger $r$ and that the Stokes layers tend to quench the convective rolls from the sidewall to smaller and smaller radii as $A$ increases. For large enough $A$, the pulsed target patterns are completely quenched at the pitchfork 
(a)

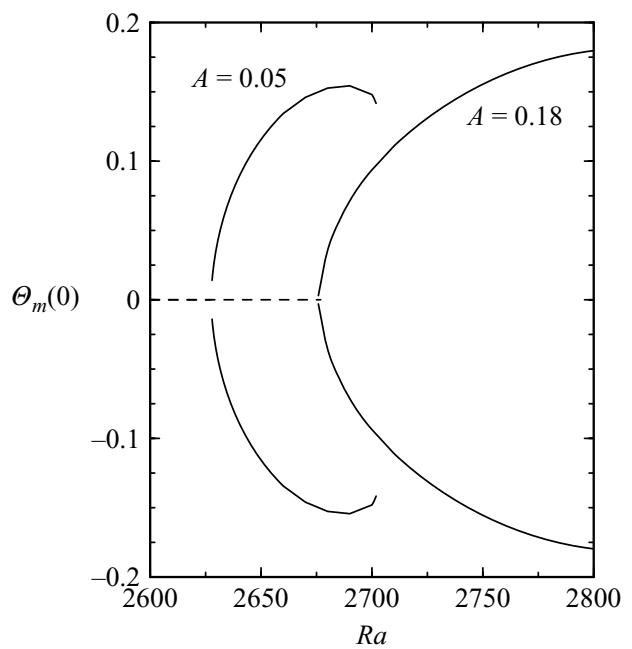

(b)

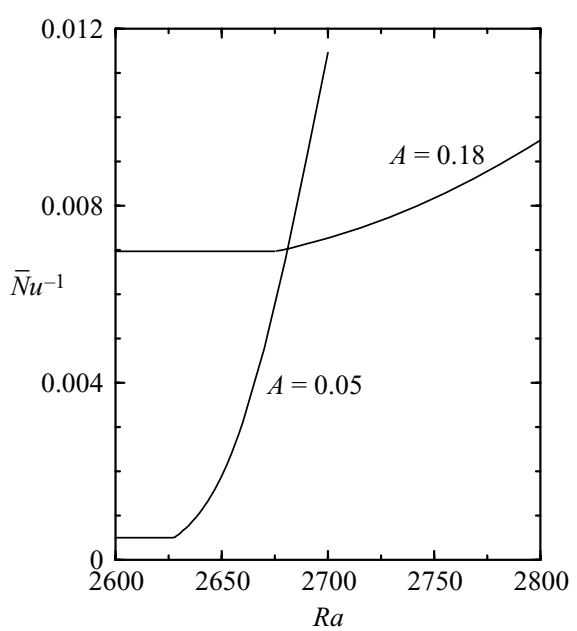

FIgURE 9. (a) Bifurcation diagrams at $\Omega_{0}=\Omega_{m}=23.6$ for $A=0.05$ and $A=0.18$, together with $(b)$ the corresponding variations of $\overline{N u}-1$ with $R a$.

(a)

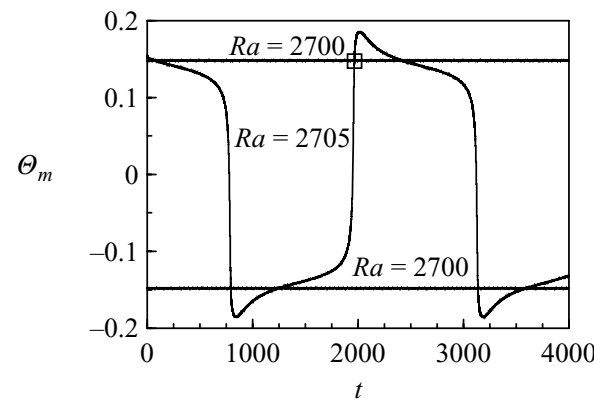

(b)

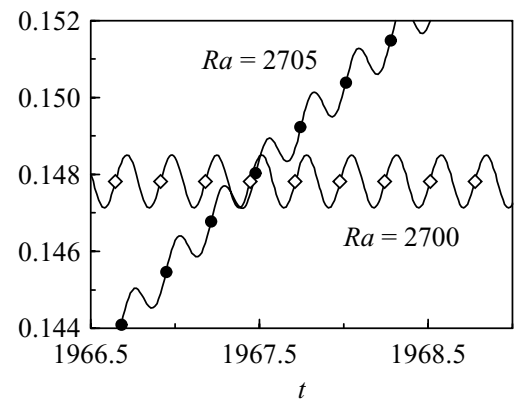

FIGURE 10. Time series of $\Theta_{m}$ at $\Omega_{0}=\Omega_{m}=23.6, A=0.05$ and $R a=2700$ and 2705. The solutions at $R a=2700$ are $K_{z}$-conjugate pulsed target patterns and the solution at 2705 is a travelling target pattern. The box where the two curves cross at about $t=1967$ is the close-up in $(b)$ which includes a Poincaré strobing $P^{n}\left(\Theta_{m}\right)=\Theta_{m}\left(n \tau_{m}\right), n$ integer, of these solutions shown as symbols superimposed on the continuous $\Theta_{m}(t)$.

bifurcation and the basic state is stabilized. In figure 8 , this stabilized basic state is shown at $A=0.2$.

The pitchfork bifurcation (using $R a$ as the bifurcation parameter) is illustrated in figure $9(a)$ for $\Omega_{0}=\Omega_{m}=23.6$ at $A=0.05$ and $A=0.18$. The measure of the flow state used in these bifurcation diagrams is $\Theta_{m}(0)$, the mid-point temperature strobed at a particular phase in the modulation (corresponding to $t=0$ ). For $R a<R a_{c}\left(A, \Omega_{0}, \Omega_{m}\right)$, the basic state (dashed line at $\Theta_{m}(0)=0$ ) is stable, and for $R a>R a_{c}$ it is unstable. At $R a=R a_{c}$, two branches of pulsed target patterns emerge at the pitchfork bifurcation as the $K_{z}$ symmetry is broken, one with a hot plume rising at the axis $\left(\Theta_{m}(0)>0\right)$ and the other with a cold plume descending at the axis $\left(\Theta_{m}(0)<0\right)$. For these states, $\Theta_{m}(t)$ has a small-amplitude oscillation synchronous with the imposed modulation, (figure 10). The close-up in figure 10(b) details this oscillation and shows the phase 
at which the solution is strobed, $P^{n}\left(\Theta_{m}\right)$, used in figure 9 and in subsequent analyses. The heat transfer properties of these states can be seen in figure $9(b)$, showing the time-averaged Nusselt number values, $\overline{N u}$, corresponding to figure $9(a)$. Notice that the $\overline{N u}$ curve changes slope (it increases discontinuously) at the bifurcation point, so the slope of the $\overline{N u}(R a)$ curve can also be used for the determination of the bifurcation point.

\subsection{Travelling target pattern: the SNIC bifurcation}

With increasing $R a$ for $A \geqslant 0.04$ or increasing $A$ for $R a \geqslant 2700$, the pulsed target patterns give way to the travelling target patterns which are distinguished by being quasi-periodic. Figure 10 shows $\Theta_{m}(t)$ for the two $K_{z}$-conjugate pulsed target patterns at $\Omega_{0}=\Omega_{m}=23.6, A=0.05$ and $R a=2700$ (approximately straight lines at $\Theta_{m} \pm 0.15$ ) and the travelling target pattern state at the same $\Omega_{0}, \Omega_{m}$ and $A$, but at slightly larger $R a=2705$ (curved line). The travelling target pattern has a very low frequency $\Omega_{w}$ in addition to the imposed modulation frequency $\Omega_{m}$. The ratio of the corresponding periods for the travelling target pattern state shown in figure $10(a)$ is $\tau_{w}: \tau_{m} \approx 8800$. The periods of these travelling wave states diverge as onset is approached from above, making it clear that the travelling target pattern solutions emerge at an infinite period bifurcation.

Strobing the travelling target patterns with the Poincare map $P$ reveals the new second period, which corresponds to the time, $\tau_{w}$, for the target pattern to repeat itself as the rolls recede in towards the axis. Figure 11 $(a)$ shows reconstructed phase portraits using delays, $\left(P^{n}\left(\Theta_{m}\right), P^{n+100}\left(\Theta_{m}\right)\right)$, of states at $\Omega_{0}=\Omega_{m}=23.6$ and $A=0.18$ at various $R a$. The basic state is a fixed point at the origin and the pulsed target patterns are symmetrically related fixed points along the diagonal (since the pulsed target pattern is a fixed point of $P$ ). The phase portrait of the travelling target pattern at $R a=2844$ consists of open circles drawn every fourth iterate of $P$ over one period $\tau_{w}$. The $\tau_{w}$-oscillation is of a slow-fast nature, where the oscillation slows significantly in the neighbourhood of the ghosts of the pulsed target patterns that were present at slightly lower $R a$. The travelling target pattern in figure 11(a) is symmetric to a half turn, indicating that the $K_{z}$-symmetry has been restored in the infinite period bifurcation (which the following results identify as a SNIC). Moreover, the travelling target pattern shows a weaker slowing down at two additional points in between the ghosts of the pulsed target patterns, and the travelling target pattern is close to being symmetric by a $90^{\circ}$ rotation. This reflects the fact that the problem has an exact $Z_{2} \times Z_{2}$ symmetry when the mean rotation is zero $\left(\Omega_{0}=0\right)$.

Figure 11(b) shows the discrete Poincare orbit $P^{n}\left(\Theta_{m}\right)$ (at the discrete times $\left.t=n \tau_{m}\right)$ for the travelling target pattern in figure 11(a), while figure 11(c) shows the corresponding $\overline{N u}-1$ time series. The period of $\overline{N u}-1$ is half the period of $P^{n}\left(\Theta_{m}\right)$. This is because the two halves of the period in $P^{n}\left(\Theta_{m}\right)$ correspond to $K_{z}$-symmetric states (see figure 13), and therefore have the same value of $\overline{N u}$, as explained in $\S 2$.

Further away from onset, the secondary oscillations in the travelling target patterns (corresponding to their radial drift) take on more of a uniform oscillator character, but this drift is still very slow compared to the imposed modulation. The spacetime diagrams in figure 12 show $P(\Theta)$ at the midplane over 2500 viscous time units corresponding to $9400 \tau_{m}$; this is equivalent to almost $9 \mathrm{~h}$ in the laboratory experiments conducted by Thompson et al. (2002). Very near the SNIC curve (case a), the travelling target pattern remains essentially unchanged for a long time (about $4500 \tau_{m}$ ) resembling one of the pulsed target patterns, then rapidly evolves to a state resembling the corresponding $K_{z}$-conjugate state. In case $b$, the state is a little further 

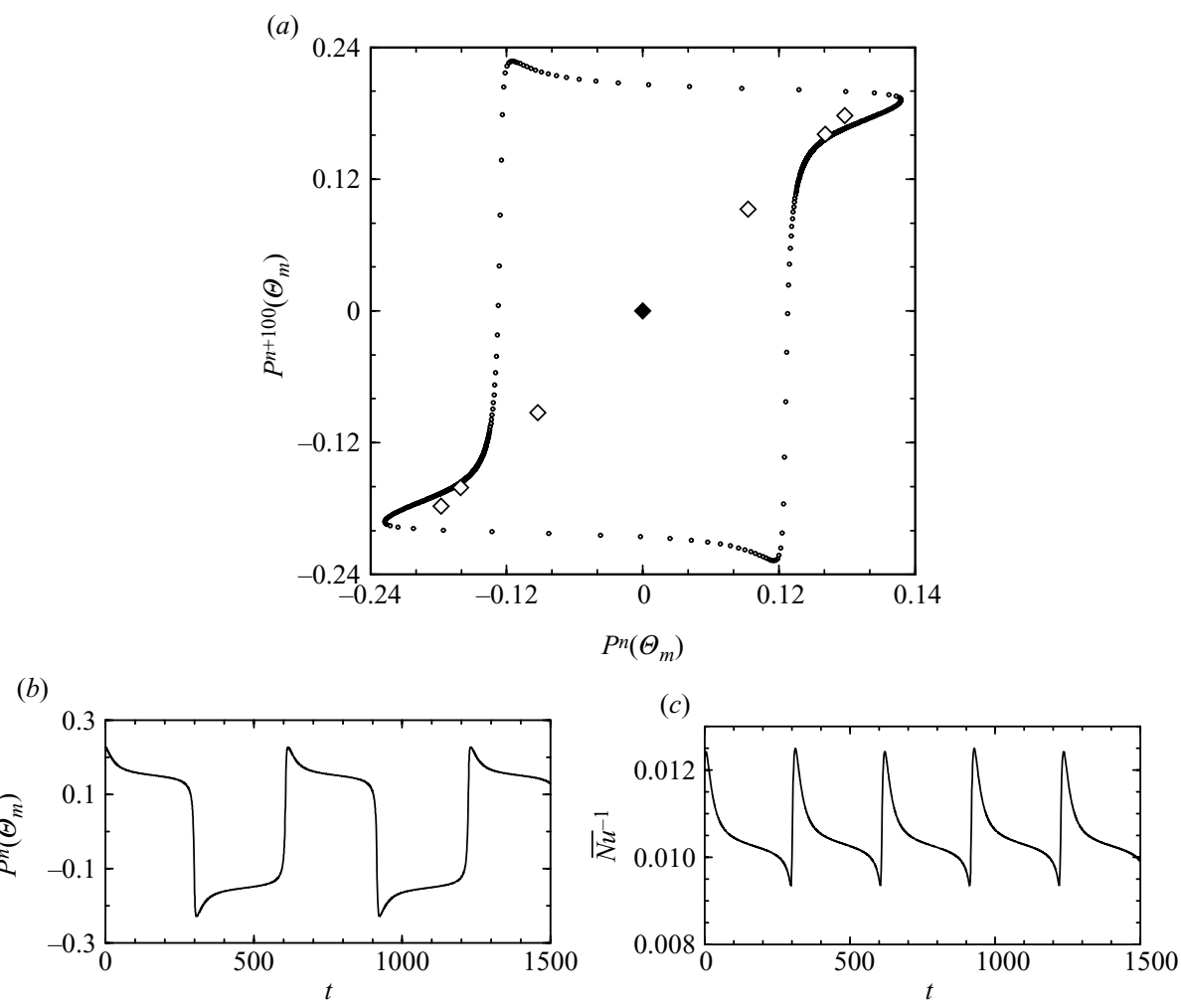

FIGURE 11. (a) Reconstructed phase portraits using delays, $\left(P^{n}\left(\Theta_{m}\right), P^{n+100}\left(\Theta_{m}\right)\right)$, of the basic state (filled diamond at the origin), pulsed target patterns (open diamonds) for $R a=2700$, 2760 and 2820, and one travelling target pattern (dots) at $R a=2844$, all for $\Omega_{0}=\Omega_{m}=23.6$ and $A=0.18$. For visual clarity only every fourth iterate of the Poincare map over one period of the travelling target pattern is shown $\left(\tau_{w}=2312 \tau_{m}\right.$ in this case). Time series of $(b) P^{n}\left(\Theta_{m}\right)$ and $(c) \overline{N u}-1$ for the travelling target pattern state in $(a)$.

removed from the SNIC curve so that the rolls drift radially inward faster, but the slow-fast characteristics are still evident. In case $c$, the state is even further away from the SNIC curve and the $\tau_{w}$ oscillations are faster and more uniform. Note that case $b$ is at relatively large $R a$ and $A$, and so the sidewall jet driven by the modulation quenches the rolls near the sidewall to a much greater extent than in case $c$ at relatively smaller $R a$ and $A$. Movies 3, 4 and 5 available with the online version of the paper show these three states over several $\tau_{w}$ periods strobed at $\tau_{m}$.

Figures 13 and 14 show eight snapshots equally spaced over the travelling-wave period $\tau_{w}$ at the same phase in the imposed $\tau_{m}$ modulation. Figure 13 shows a large $A=0.18$ case in which the strong Stokes layers have suppressed the convection rolls away from the axis region, and the modulation-driven cells at the corners provide non-trivial heat exchange at the sidewall. This is the same solution as shown in figure 12(b). Far from the axis there are weak concentric ring plumes that emerge up to several $d$ from the sidewall, gaining strength as they drift toward the axis. At the axis, the innermost plume weakens and disappears entirely before being replaced by the adjacent roll. Figure 14 shows an $A=0.05$ case in which the travelling target pattern fills the whole cell. This is the same solution as shown in figure 12(a). The sidewall jet perturbs the nearby velocity and temperature fields, resulting in small inward and 
(a)

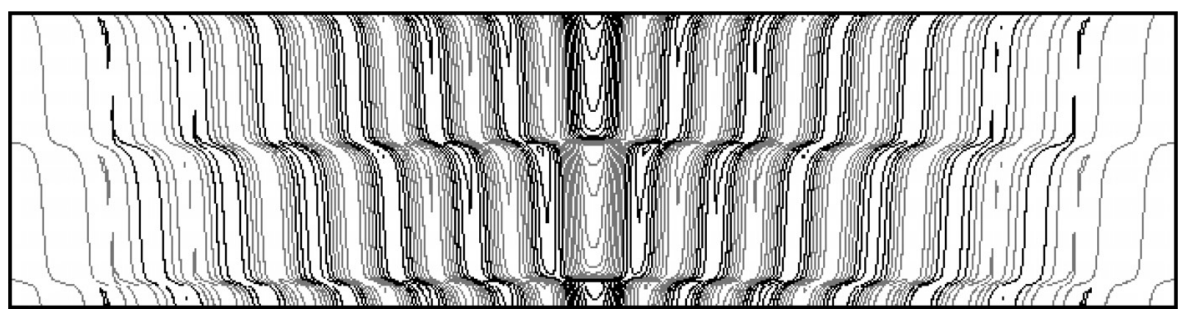

(b)

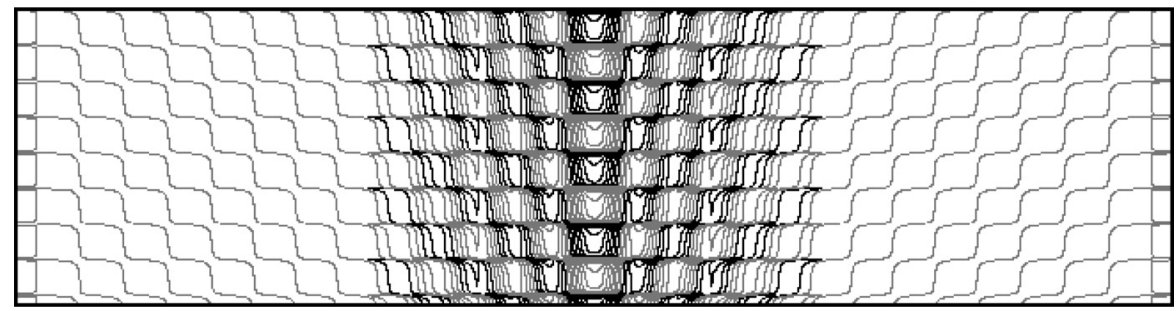

(c)

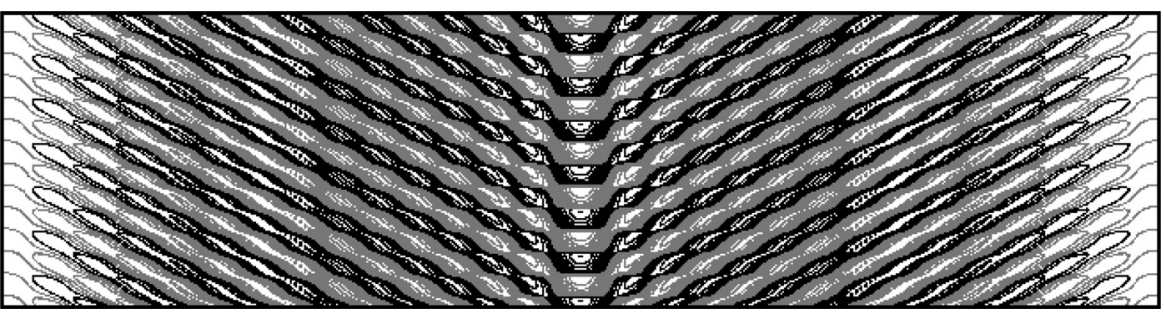

FIGURE 12. Space-time plot of travelling target states at $\Omega_{0}=\Omega_{m}=23.6$ for various $R a$ and $A$, showing $\Theta$ at $z=0$ with time running from top to bottom over 2500 viscous times. $(a)$ $R a=2705$ and $A=0.05$; (b) $R a=2844$ and $A=0.18$; (c) $R a=2840$ and $A=0.06$. Movies 3,4 and 5 show these three states over several $\tau_{w}$ periods strobed at $\tau_{m}$.

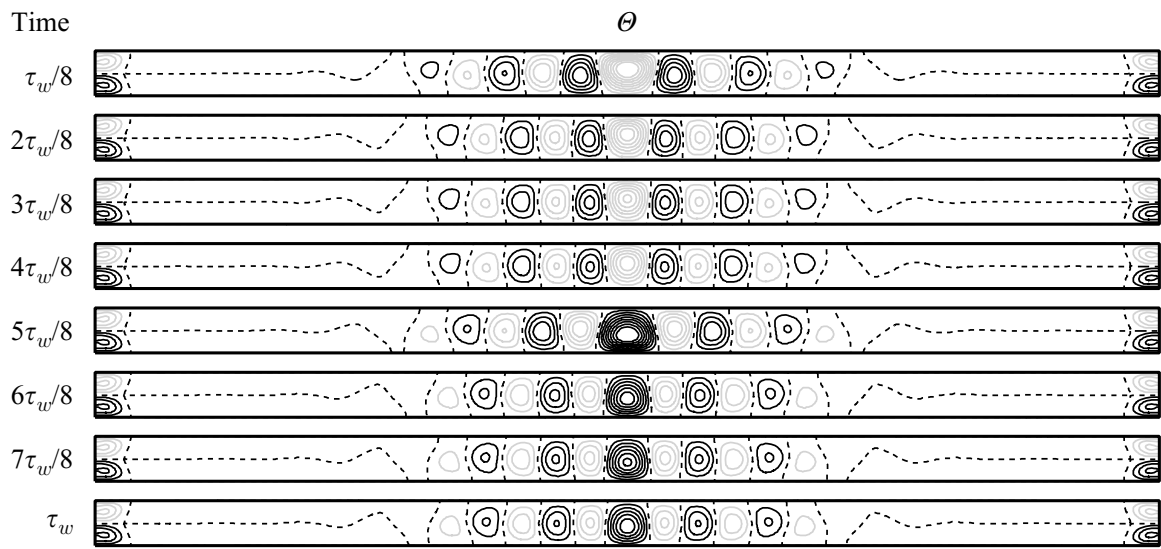

Figure 13. Contours of temperature deviation in a meridional plane $(r \in[-\gamma, \gamma], z \in$ $[-0.5,0.5])$ over one period of the travelling target pattern at $R a=2844, \Omega_{0}=\Omega_{m}=23.6$ and $A=0.18$, the same solution as shown in figure $12(b)$. Five contours are shown linearly spaced between -0.2 and 0.2 .

outward motions of the outermost cells as well as a mechanically driven heat transport. Both take place on the $\tau_{m}$ time scale, as do those in the nearby pulsed target pattern (figure 4). Close-ups of the radial velocity and $\Theta$ near the sidewall region for this case are shown in figure 15. The sequence shown in figure 15 is over the much slower $\tau_{w}$ time 
Time

$\Theta$

$\tau_{w} / 8$ E: $:$ :

$2 \tau_{w} / 8$ ㄴ:

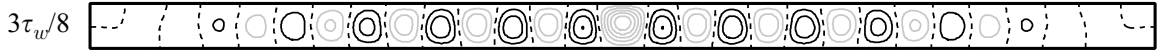

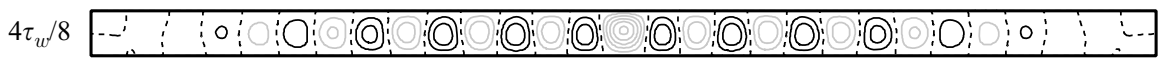

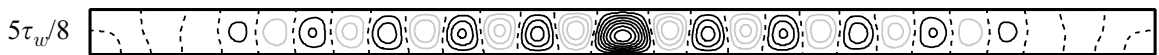

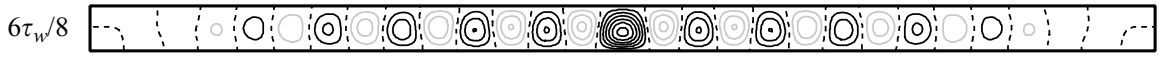

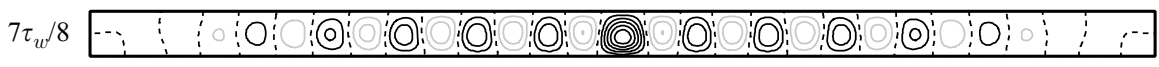

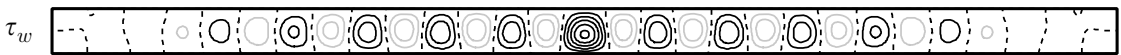

Figure 14. Contours of temperature deviation in a meridional plane $(r \in[-\gamma, \gamma], z \in$ $[-0.5,0.5])$ over one period of the travelling target pattern at $R a=2705, \Omega_{0}=\Omega_{m}=23.6$ and $A=0.05$, the same solution as shown in figure $12(a)$. Eight contours are shown linearly spaced between -0.2 and 0.2 .

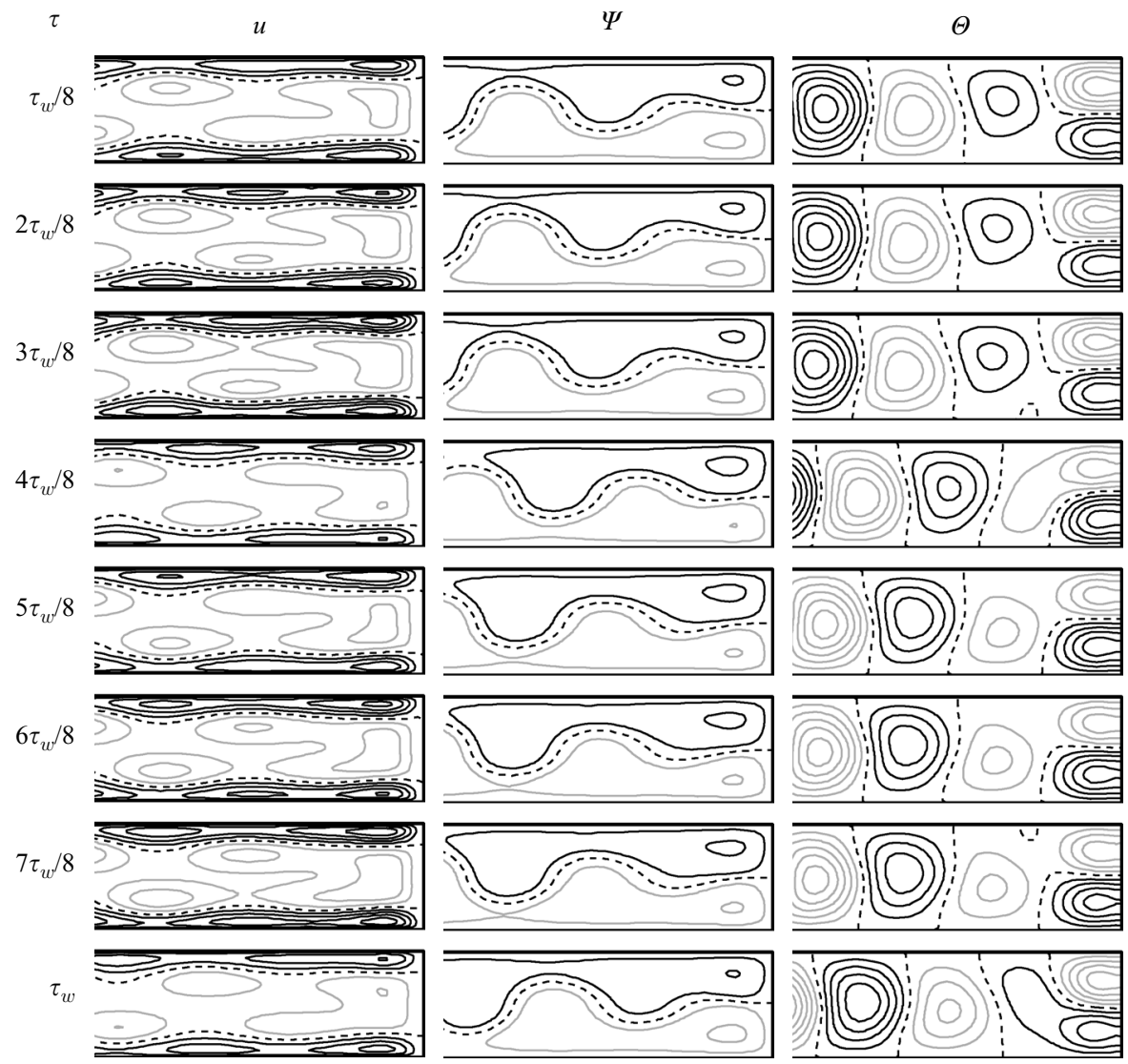

FIGURE 15. Snapshots of $u, \Psi$ and $\Theta$, all at the same phase of the imposed modulation in a meridional plane $(r \in[0.74 \gamma, \gamma], z \in[-0.5,0.5])$ over one period $\tau_{w}$ at $R a=2705$, $\Omega_{0}=\Omega_{m}=23.6$ and $A=0.05$. Five contours are quadratically spaced for $\Psi \in[-0.25,0.25]$ and linearly spaced for $\Theta \in[-0.025,0.025]$ and $u \in[-10,10]$. 


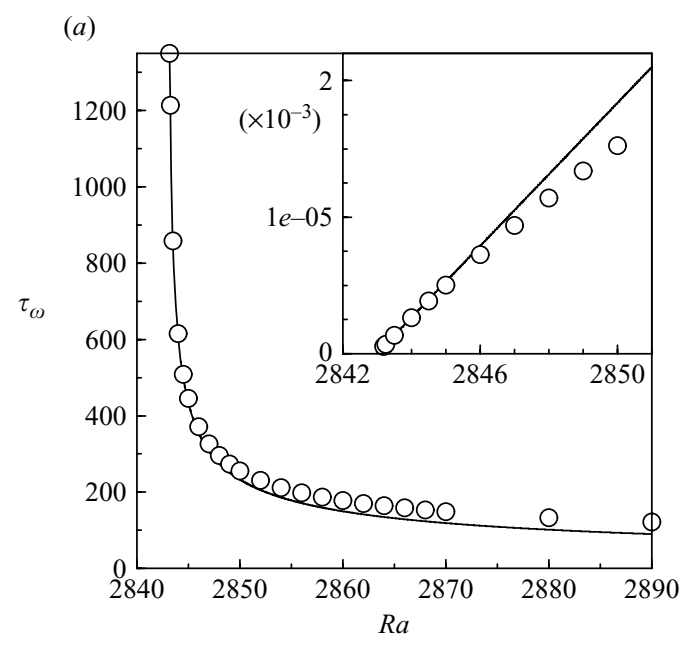

(b)

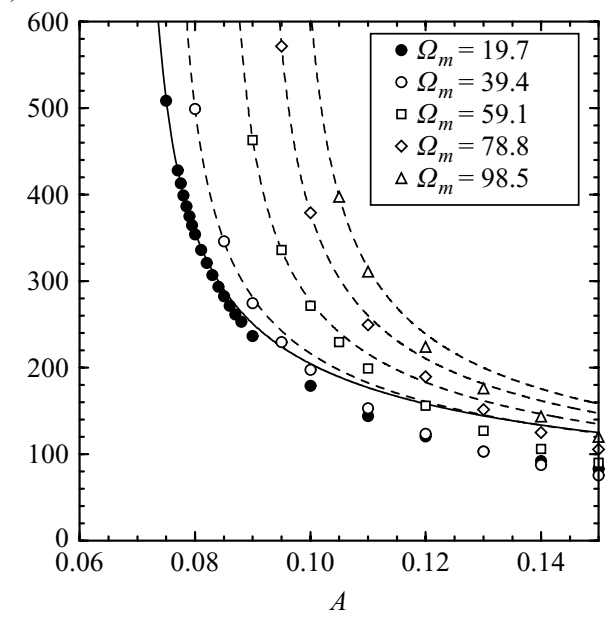

FiguRE 16. Variation of $\tau_{w}(a)$ with $R a$ for $A=0.18$ and $\Omega_{0}=\Omega_{m}=23.6$, and $(b)$ with $A$ for $R a=2958, \Omega_{0}=19.7$ and $\Omega_{m}$ as indicated. The symbols are numerical estimates and the curves are fits to the first four data points for each $\Omega_{m}$ set of the form $\tau_{w}=a / \sqrt{\left(R a-R a_{S N I C}\right)}$. The inset in $(a)$ is $\tau_{w}^{-2}(R a)$.

scale $\left(\tau_{w} \approx 8800 \tau_{m}\right.$ ), and each frame is taken at the same phase in the $\tau_{m}$ modulation. This is why the relatively hot (cold) cell is always at the top (bottom) corner in these figures. During the $\tau_{w}$ cycle, the concentric rolls making up the target pattern drift radially inward. A new roll is formed as the void left by the outermost receding roll is filled by relatively warm (cool) fluid seeping out from the bottom (top) corner cell each half $\tau_{w}$ period. The modulation-driven Stokes layers and the corresponding midplane jet (all characterized by the radial velocity) do not appear to play a significant role in the drift dynamics at the $\tau_{w}$ time scale, although their action on the $\tau_{m}$ time scale continues to be essentially the same as for the pulsed target patterns described earlier.

Figure 16(a) shows the variation of $\tau_{w}$ for different $R a$ with $A=0.18$ and $\Omega_{0}=$ $\Omega_{m}=23.6$. As $R a \rightarrow R a_{S N I C}$ from above, $\tau_{w} \rightarrow \infty$ following the $1 / \sqrt{\left(R a-R a_{S N I C}\right)}$ scaling associated with the saddle-node on an invariant circle (SNIC) bifurcation. Figure 16(b) shows the variation of $\tau_{w}$ with respect to $A$ for $R a=2958, \Omega_{0}=19.7$ and various $\Omega_{m}$.

In general, a SNIC bifurcation consists of a saddle-node bifurcation taking place on a limit cycle, as shown schematically in figure 17(a). Before the bifurcation (lefthand diagram), the period of the limit cycle tends to infinity as the bifurcation is approached, and the periodic solution spends more and more time near the place where the saddle-node will appear. The periodic solution becomes a homoclinic orbit at the bifurcation point (central diagram). After the bifurcation, the periodic solution no longer exists and there remains a pair of fixed points, one a saddle (unstable, hollow in the figure) and the other a node (stable, solid in the figure), and stable and unstable manifolds connecting them. This is a codimension-one bifurcation (Kuznetsov 2004). In the presence of a $Z_{2}$ symmetry, if the limit cycle is not pointwise $Z_{2}$-invariant, a pair of saddle-nodes appear simultaneously on the invariant circle (figure 17b). This is what happens in our problem, but instead of a limit cycle we have quasi-periodic solutions (a two-torus) (figure 17c). In this case, the two saddlenodes become saddle-nodes of limit cycles; the two $Z_{2}$-symmetric saddle-nodes at the 
(a)

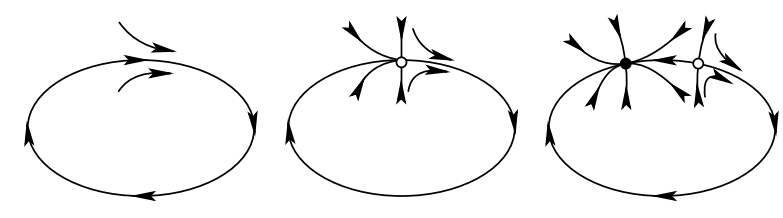

(c)

(b)
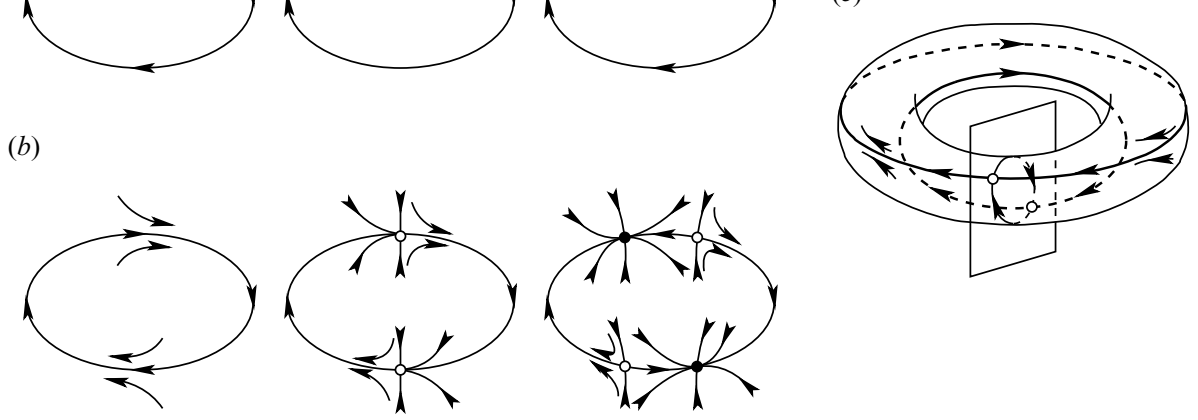

FIGURE 17. Schematic of the SNIC bifurcation on an invariant circle in $(a)$ a generic system, and $(b)$ a $Z_{2}$-symmetric system; left, central and right diagrams correspond to before, during and after the SNIC bifurcation. (c) Schematic of the SNIC bifurcation on an invariant two-torus; the planar section (a Poincaré section) corresponds to case $(b)$ centre.

bifurcation point are shown as thick lines. Analogous $Z_{2}$-symmetry breaking between rotating and modulated rotating waves in Taylor-Couette flow via a SNIC bifurcation has been computed and observed experimentally (Abshagen et al. 2005a,b). In the Poincaré section of a quasi-periodic solution (the planar section of the two-torus in figure $17 c$ ), the bifurcation occurs as a $Z_{2}$-symmetric collision of saddle-nodes shown in figure $17(b)$. The invariant circle $\mathscr{C}$ on the Poincare section is $K_{z}$ invariant as a set; i.e. given a point $a \in \mathscr{C}, P a \in \mathscr{C}$ although $a \neq P a$. The continuous quasi-periodic solution starting at $a$ becomes a discrete orbit on $\mathscr{C}$ made of the iterates of $a$ under $P\left(a, P a, P^{2} a, P^{3} a, \ldots\right)$. This discrete orbit densely fills $\mathscr{C}$ when $\tau_{w} / \tau_{m}$ is not rational (the generic case) and in this case, the $K_{z}$ reflections of the iterates do not belong to the discrete orbit, i.e. the discrete orbit is not $K_{z}$ invariant. This is clearly illustrated in figure 11( $a$ ), where we have plotted every fourth iterate of $P$ over one period $\tau_{w}$. These iterates are on the invariant circle $\mathscr{C}$, and almost fill it near the ghosts of the saddle nodes. If we keep iterating $P$, the result would be the invariant circle $\mathscr{C}$.

When $\tau_{w} / \tau_{m}=p / q$ is rational, the solution is strictly periodic, of period $q \tau_{w}=p \tau_{m}$, and if $p$ is even, the discrete orbit is $K_{z}$ invariant, i.e. applying $K_{z}$ is the same as advancing in time by the half-period $\tau_{w} / 2$. Therefore, in this case, the solution is invariant under a space-time symmetry consisting of applying $K_{z}$ and advancing in time by half the travelling-wave period. In the case illustrated in figure $11(a)$, there are approximately 6000 iterates of $P$ in a travelling-wave period $\tau_{w}$, and so $K_{z} a$ plus a $\tau_{w} / 2$ advance in time is very close to an iterate of $P$, and the space-time symmetry is very nearly satisfied, as can be seen in figures 12,14 and 15 .

In the continuous time system, the bifurcation occurs as a $Z_{2}$-symmetry-restoring SNIC on an invariant two-torus, as illustrated in figure $17(c)$. By measuring $\tau_{w}$ near onset for assorted values of $(R a, A)$ and applying the scaling law, the location of the SNIC bifurcation curve shown in figure 18 was estimated. To the left of the pitchfork bifurcation curve (dark grey in the figure), the basic state is stable, to the right (white in the figure) the pair of $K_{z}$-conjugate pulsed target patterns are stable, and beyond the SNIC bifurcation curve (light grey in the figure) the travelling target pattern is stable. 


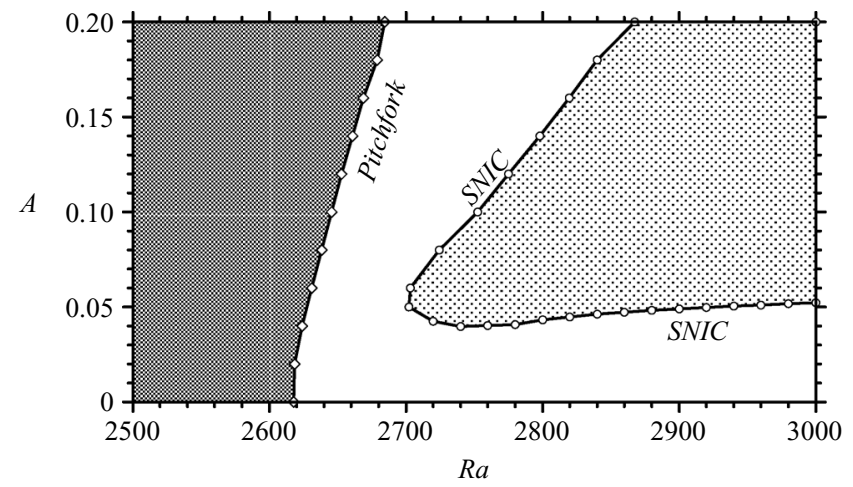

FIGURE 18. Regime diagram for $\Omega_{0}=\Omega_{m}=23.6$, showing the pitchfork and SNIC bifurcation curves. States in the different regions: modulated basic state (dark grey), pulsed target patterns (white), and travelling target patterns (light grey).

\section{Discussion and conclusions}

The spatio-temporal chaos associated with Küppers-Lortz instability in rotating convection can be greatly mitigated by small-amplitude high-frequency modulations of the rotation rate, as observed experimentally by Thompson et al. (2002). In this paper, we have investigated the responsible mechanisms by employing simulations of the Navier-Stokes-Boussinesq equations with the corresponding physical boundary conditions, and analysed the nonlinear solutions with the aid of equivariant bifurcation theory. The imposed modulation drives Stokes layers at the top and bottom endplates of the cylinder. The flow in these Stokes layers is fed into the interior by the presence of the cylinder sidewall, where a radial jet forms at mid-height. These oscillatory Stokes layers and jet tend to quench instabilities to three-dimensional flow, and as observed experimentally, axisymmetric target pattern states result. The main dynamic role of the modulation is to drive the system toward the axisymmetric subspace, but the subsequent dynamics of the axisymmetric states is apparently decoupled from the modulations. The use of a stroboscopic map restricts the analysis of the dynamics to a Poincare section which is transverse to the modulations and provides a clearer picture of the dynamics. In the Poincare section, the modulated basic state is a $Z_{2}$ reflection symmetric fixed point that first loses stability at a pitchfork bifurcation, breaking the $Z_{2}$ symmetry, where a pair of pulsed target pattern states are born. These are also fixed points in the Poincare section and are $Z_{2}$ reflections of each other. They lose stability at a saddle-node on an invariant circle (SNIC) bifurcation, which results in the birth of the travelling target pattern state that motivates this study. The new frequency introduced by this bifurcation goes to zero at onset and corresponds to the time scale associated with the slow drift of the concentric rolls radially inward. After the SNIC bifurcation, the quasi-periodic solution (travelling target pattern) lives on a two-torus, which is $Z_{2}$ reflection symmetric. The Poincaré section of the two-torus results in an invariant circle $\mathscr{C}$, which is also $Z_{2}$ reflection symmetric. The travelling target pattern becomes a discrete orbit on the invariant circle, densely filling it (except when the two frequencies are commensurate). As the two-torus and the invariant circle $\mathscr{C}$ are $K_{z}$ reflection symmetric, the SNIC bifurcation is a symmetry-restoring bifurcation. However, individual solutions are not $K_{z}$-symmetric, but in many cases, and in particular close to the SNIC bifurcation, the $K_{z}$-symmetry is very approximately satisfied, taking the form of a space-time 
symmetry: advancing half the travelling-wave period and applying the $K_{z}$-symmetry leaves the solution invariant.

The mechanism for the onset of the travelling target patterns was not identified in the experimental study (Thompson et al. 2002) and the Swift-Hohenberg (SH) amplitude equations model (Roxin \& Riecke 2002) was unable to reproduce such travelling target patterns. They surmised that their model equations lacked some non-variational terms to permit such persistent dynamics. This SH model is twodimensional in $(r, \theta)$, and since the $z$-coordinate is absent, the model lacks any vertical information. By solving the full problem described by the Navier-Stokes equations incorporating the Boussinesq approximation, we were able to reproduce the travelling target patterns in the parameter regimes where they were observed experimentally, and having the same spatio-temporal characteristics as those observed experimentally. Our analysis of these solutions indicates that the breaking of the socalled Boussinesq symmetry (a $Z_{2}$ reflection symmetry) plays a crucial role in the drift dynamics. Roxin \& Riecke (2002) included odd terms in their SH model in order to impose a generic $Z_{2}$ invariance in their amplitude equation, which they claimed mimicked the Boussinesq symmetry. However, the amplitude equations lack any vertical information (the spatial differential operators are only horizontal), and the $Z_{2}$ invariance imposed in the SH model cannot have any relationship with the vertical reflection $K_{z}(z \rightarrow-z)$. Furthermore, we have found that the transition to the travelling target patterns is a symmetry-restoring SNIC bifurcation where the resultant travelling target pattern inherits a spatio-temporal $Z_{2}$ symmetry from the pair of $Z_{2}$-related asymmetric pulsed target patterns which are annihilated at the SNIC bifurcation. So even though it is tempting to think of these states as essentially two-dimensional (horizontal $(r, \theta)$ patterns), their structure in the vertical direction is crucial in determining their dynamics. In particular, the manner in which the Boussinesq symmetry is broken and then restored as a spatio-temporal symmetry holds the key to understanding the onset of the travelling target patterns.

In the physical experiments (Thompson et al. 2002) and in the Swift-Hohenberg amplitude equations model (Roxin \& Riecke 2002) of the modulated rotating convection problem, when the initial condition before the modulation is imposed was a KL state, the modulations generically result in imperfect spiral patterns. In order to obtain axisymmetric target patterns, both the experiments and the $\mathrm{SH}$ model needed to generate these 'with care' using convoluted initial conditions, etc. However, once a target pattern was obtained, it persisted for long times. Our results are consistent with these observations, in that if we start with a KL state and then start modulating the rotation, the flow evolves to an imperfect spiral state. When we computed in the axisymmetric subspace, however, the target patterns are obtained. If we add a small non-axisymmetric perturbation to the target patterns it takes a long time (depending on the magnitude of the perturbation) for the perturbation to grow, but it does grow. Our preliminary indications are that the target patterns may not be stable to threedimensional perturbations. In fact, in the limit of vanishing modulation $(A \rightarrow 0)$, the stable state is the three-dimensional KL state. Therefore if the target patterns are stable when $A \neq 0$, there must be a critical $A$ below which the target patterns are unstable. To address the stability of the target patterns to three-dimensional perturbations is not trivial. We must compute the time-periodic axisymmetric target pattern state (which we have done in this paper) and then perform a Floquet stability analysis to general three-dimensional perturbations, which is beyond the scope of this paper. Very closely related is the question of whether the spiral patterns or the target patterns bifurcate first from the modulated basic state as $R a$ is increased, for a given modulation $A \neq 0$. 
In summary, the effects of harmonic modulations on rotating convection have been investigated numerically, and the numerical solutions have been analysed using bifurcation-theoretical techniques and in particular equivariant dynamical systems theory. The role of symmetry-breaking in the SNIC bifurcation is central to understanding the onset of the travelling target patterns, a phenomenon which had remained completely unexplained up to now.

We thank O. Batiste and I. Mercader for providing us with the first version of the numerical codes used in this project, and for stimulating discussions on the physics and numerics of this problem. This work was supported by the National Science Foundation grant DMS-0505489, the Spanish Ministry of Education and Science grant FIS2004-01336, Catalonian Government grant SGR-00024, and a fellowship through the Alfred P. Sloan Foundation. Part of this work was completed while A. R. was a Fulbright graduate fellow at UPC. Computational resources of ASU's Fulton HPCI are greatly appreciated.

\section{REFERENCES}

Abshagen, J., Lopez, J. M., Marques, F. \& Pfister, G. 2005 a Mode competition of rotating waves in reflection-symmetric Taylor-Couette flow. J. Fluid Mech. 540, 269-299.

Abshagen, J., Lopez, J. M., Marques, F. \& Pfister, G. 2005b Symmetry breaking via global bifurcations of modulated rotating waves in hydrodynamics. Phys. Rev. Lett. 94, 074101.

Avila, M., Marques, F., Lopez, J. M. \& Meseguer, A. 2007 Stability control and catastrophic transition in a forced Taylor-Couette system. J. Fluid Mech. 590, 471-496.

Batchelor, G. K. 1967 An Introduction to Fluid Dynamics. Cambridge University Press.

Becker, N., Scheel, J. D., Cross, M. C. \& Ahlers, G. 2006 Effect of the centrifugal force on domain chaos in Rayleigh-Bénard convection. Phys. Rev. E 73, 066309.

BhattacharJeE, J. K. 1990 Convective instability in a rotating fluid layer under modulation of the rotating rate. Phys. Rev. A 41, 5491-5494.

Bodenschatz, E., Pesch, W. \& Ahlers, G. 2000 Recent developments in Rayleigh-Bénard convection. Annu. Rev. Fluid Mech. 32, 709-778.

Davis, S. H. 1976 The stability of time-periodic flows. Annu. Rev. Fluid Mech. 8, 57-74.

Fornberg, B. 1998 A Practical Guide to Pseudospectral Methods. Cambridge University Press.

Hugues, S. \& Randriamampianina, A. 1998 An improved projection scheme applied to pseudospectral methods for the incompressible Navier-Stokes equations. Intl J. Numer. Meth. Fluids 28, 501-521.

yon KerczeK, C. \& Davis, S. H. 1974 Linear stability theory of oscillatory Stokes layers. J. Fluid Mech. 62, 753-773.

KNobloch, E. 1998 Rotating convection: recent developments. Intl J.Engng Sci. 36, 1421-1450.

KüPPERS, G. \& LORTZ, D. 1969 Transition from laminar convection to thermal turbulence in a rotating fluid layer. J. Fluid Mech. 35, 609-620.

KuZnetsov, Y. A. 2004 Elements of Applied Bifurcation Theory, 3rd edn. Springer.

Lopez, J. M., Rubio, A. \& Marques, F. 2006 Travelling circular waves in axisymmetric rotating convection. J. Fluid Mech. 569, 331-348.

Lopez, J. M., Marques, F., Mercader, I. \& Batiste, O. 2007 Onset of convection in a moderate aspect-ratio rotating cylinder: Eckhaus-Benjamin-Feir instability. J. Fluid Mech. 590, 187-208.

Lopez, J. M., CUI, Y. D., MARQues, F. \& LiM, T. T. 2008 Quenching of vortex breakdown oscillations via harmonic modulation. J. Fluid Mech. 599, 441-464.

MArques, F. \& Lopez, J. M. 1997 Taylor-Couette flow with axial oscillations of the inner cylinder: Floquet analysis of the basic flow. J. Fluid Mech. 348, 153-175.

Marques, F., Mercader, I., Batiste, O. \& Lopez, J. M. 2007 Centrifugal effects in rotating convection: axisymmetric states and three-dimensional instabilities. J. Fluid Mech. 580, 303318. 
Mercader, I., Net, M. \& Falqués, A. 1991 Spectral methods for high order equations. Comput. Meth. Appl. Mech. Engng 91, 1245-1251.

Niemela, J. J., Smith, M. R. \& Donnelly, R. J. 1991 Convective instability with time-varying rotation. Phys. Rev. A 44, 8406-8409.

Orszag, S. A. \& Patera, A. T. 1983 Secondary instability of wall-bounded shear flows. J. Fluid Mech. 128, 347-385.

Roppo, M. N., Davis, S. H. \& Rosenblat, S. 1984 Bénard convection with time-periodic heating. Phys. Fluids 27, 796-803.

Roxin, A. \& Riecke, H. 2002 Rotating convection in an anisotropic system. Phys. Rev. E 65, 046219.

Sánchez-Álvarez, J. J., Serre, E., Crespo del Arco, E. \& Busse, F. H. 2005 Square patterns in rotating Rayleigh-Bénard convection. Phys. Rev. E 036307.

Scheel, J. D. \& Cross, M. C. 2005 Scaling laws for rotating Rayleigh-Bénard convection. Phys. Rev. E 72, 056315.

Sinha, M., Kevrekidis, I. G. \& Smits, A. J. 2006 Experimental study of a Neimark-Sacker bifurcation in axially forced Taylor-Couette flow. J. Fluid Mech. 558, 1-32.

Stokes, G. G. 1851 On the effect of the internal friction of fluids on the motion of pendulums. Trans. Camb. Phil. Soc. 9, 8.

Thompson, K. L., BajaJ, K. M. S. \& Ahlers, G. 2002 Travelling concentric-roll patterns in Rayleigh-Bénard convection with modulated rotation. Phys. Rev. E 65, 046218.

Tuckerman, L. S. \& BARKLeY, D. 1988 Global bifurcation to travelling waves in axisymmetric convection. Phys. Rev. Lett. 61, 408-411.

Weisberg, A. Y., Kevrekidis, I. G. \& Smits, A. J. 1997 Delaying transition in Taylor-Couette flow with axial motion of the inner cylinder. J. Fluid Mech. 348, 141-151.

Yıн, C.-S. 1977 Fluid Mechanics. West River Press, Ann Arbor, Michigan. 